\title{
ON MODIFICATION THEOREMS
}

\author{
BY \\ MURALI RAO
}

\begin{abstract}
Given a right continuous family $F_{t}$ of complete $\sigma$-fields and a bounded right continuous family $X_{t}$ of random variables, we show in this paper that it is possible to modify the conditional expectations $E\left(X_{t} \mid F_{t}\right)$ to be right continuous. When $X_{t}=X$, this reduces to a result of $\mathbf{J}$. L. Doob.
\end{abstract}

A famous result of Doob states that any martingale has a right continuous with left limits modification. This is an important result and is very useful in providing modification theorems in the theory of Markov processes.

During a discussion with Professor T. Watanabe the following problem arose:

Suppose $F_{t}$ is an increasing right continuous family of $\sigma$-fields and $X_{t}$ is a right continuous with left limits stochastic process. Can we define a right continuous with left limits modification of $E\left(X_{t} \mid F_{t}\right)$ ?

We shall show that it is always possible to select such a modification. Note that whereas in the case of martingales we have available the upcrossing inequality of Doob (this is an indispensable tool in the standard proofs of the martingale modification theorem) there is no such tool in the general situation. The method developed here generalizes without changes to Banach space valued martingales. Thus a Banach space valued martingale has a right continuous with left limits modification. Our methods do not require the notion of separability. The only prerequisite to reading this note is the knowledge of the martingale convergence theorem and familiarity with the notion of stopping rules. In the beginning of $\S 1$ we develop what is needed about stopping rules in a more general setting than what is given in standard books. Then we proceed to prove the main theorem (Theorem 6).

In $\S 2$ we show how the modification theorem for super martingales can be deduced using Theorem 6. Lemma 9 gives a slight generalization of a theorem of Meyer without invoking the upcrossing inequality.

Thanks are due to Karl Pedersen for many valuable discussions.

1. Let $F_{n}$ denote a sequence of $\sigma$-fields for $n=1,2, \ldots, \infty ; \infty$ is included. An integral valued nonnegative function $T$ is called a stopping rule relative to $F_{n}$ iff for all $n$ the event $(T=n) \in F_{n}$. If $T$ is a stopping rule we denote by $F_{T}$ the $\sigma$-fields of events $A$ such that $A \cap(T=n) \in F_{n}, 1 \leqq n \leqq \infty$. It is easy to verify that $F_{T}$ is indeed a $\sigma$-field. We simply note the following:

1. $T$ is $F_{T}$-measurable.

Received by the editors November 11, 1970.

AMS 1970 subject classifications. Primary 60GXX.

Key words and phrases. Stochastic process, conditional expectation, modification, stopping times, super martingales.

Copyright (c) 1972, American Mathematical Society 
2. If $X_{n}$ is a sequence of random variables with $X_{n} F_{n}$-measurable for $1 \leqq n \leqq \infty$ then $X_{T}$ is $F_{T}$-measurable.

3. Suppose the $\sigma$-fields $F_{n}$ increase, i.e. $F_{n} \subset F_{n+1}$. Then if $T, S$ are stopping rules and $T \leqq S$ we have $F_{T} \subset F_{S}$. If on the other hand the $\sigma$-fields decrease and $T, S$ are stopping rules with $T \leqq S$ then $F_{T} \supset F_{S}$.

The following lemma has an easy proof.

Lemma 1 (Optional SAMPLING Lemma). Let $X_{n}$ be random variables and $F_{n}$ $\sigma$-fields for $1 \leqq n \leqq \infty$. We will assume that all expectations occurring exist. Let $T$ be a stopping rule. If $Y_{n}=E\left(X_{n} \mid F_{n}\right), 1 \leqq n \leqq \infty$, then $Y_{T}=E\left(X_{T} \mid F_{T}\right)$ almost surely.

The proof is easy. In fact $Y_{T}$ is $F_{T}$-measurable. Hence the result follows from the equalities

$$
\int_{A \cap(T=n)} X_{T}=\int_{A \cap(T=n)} X_{n}=\int_{A \cap(T=n)} Y_{n}=\int_{A \cap(T=n)} Y_{T}
$$

and now sum over $n ; A$ denotes an arbitrary element of $F_{T}$.

REMARK. If for all $n, X_{n}=X$ and the $\sigma$-fields $F_{n}$ increase or decrease, Lemma 1 is the optional sampling theorem for martingales. The general optional sampling theorem for super martingales then follows at once via the Doob decomposition $[1$, p. 104].

LEMMA 2. Let $F_{n}$ be an increasing sequence of $\sigma$-fields; $1 \leqq n \leqq \infty$. Let $X_{n}, 1 \leqq n \leqq \infty$, be a sequence of random variables such that

(1) $X_{n} \rightarrow X_{\infty}$ almost surely,

(2) $\left|X_{n}\right| \leqq \varphi$ with $E(\varphi)<\infty$.

Then $\left|E\left(X_{n} \mid F_{n}\right)-E\left(X_{\infty} \mid F_{n}\right)\right| \rightarrow 0$ almost surely.

Proof. We may assume that $X_{\infty}=0$. Put $Y_{n}=E\left(X_{n} \mid F_{n}\right)$. We shall show that $Y_{n} \rightarrow 0$ a.s. Let $\varepsilon>0$. Define the stopping rules $T_{k}$ by

$$
\begin{aligned}
T_{1} & =\inf \left(n:\left|Y_{n}\right| \geqq \varepsilon\right), & T_{k+1} & =\inf \left(n: n \geqq T_{k}+1,\left|Y_{n}\right| \geqq \varepsilon\right), \\
& =\infty \text { if there is no such } n ; & & =\infty \text { if there is no such } n .
\end{aligned}
$$

Then $1+T_{k} \leqq T_{k+1}, T_{k}<\infty$ implies $\left|Y_{T_{k}}\right| \geqq \varepsilon$, and if $T_{k}=\infty$ then $\left|Y_{T_{k}}\right|=0$. We have

$$
\varepsilon P\left(T_{k}<\infty\right) \leqq E\left(\left|Y_{T_{k}}\right|\right) \leqq E\left(\left|X_{T_{k}}\right|\right)
$$

because $Y_{T_{k}}=E\left(X_{T_{k}} \mid F_{T_{k}}\right)$. Since $T_{k} \geqq k$ by definition (note that $T_{1} \geqq 1$ ), $T_{k} \rightarrow \infty$, and hence $X_{T_{k}} \rightarrow 0$. Therefore $\left(E\left|X_{T_{k}}\right|\right)$ and hence $P\left(T_{k}<\infty\right)$ tend to zero as $k \rightarrow \infty$. Also (lim sup $\left.\left|Y_{n}\right| \geqq \varepsilon\right)=\left(T_{k}<\infty\right.$ for all $\left.k\right)=\bigcap_{k}\left(T_{k}<\infty\right)$. Hence $P\left(\lim \sup \left|Y_{n}\right| \geqq \varepsilon\right)$ $\leqq \lim \inf P\left(T_{k}<\infty\right)=0$. Q.E.D.

A version of Lemma 2 for decreasing $\sigma$-fields also holds. We have

Lemma 3. Let $F_{n}$ be a decreasing sequence of $\sigma$-fields. Let $X_{n}, 1 \leqq n \leqq \infty$, be $a$ sequence of random variables with

(1) $X_{n} \rightarrow X_{\infty}$ almost surely,

(2) $\left|X_{n}\right| \leqq \varphi$ with $E(\varphi)<\infty$.

Then $\left|E\left(X_{n} \mid F_{n}\right)-E\left(X_{\infty} \mid F_{n}\right)\right| \rightarrow 0$ almost surely. 
Proof. Assume that $X_{\infty}=0$ and put $Y_{n}=E\left(X_{n} \mid F_{n}\right)$. Let $\varepsilon>0$ and define the stopping rules $T_{n}$ by

$$
\begin{aligned}
T_{n} & =\sup \left(k: k \leqq n \text { and }\left|Y_{k}\right| \geqq \varepsilon\right), \\
& =1 \quad \text { if there is no such } k .
\end{aligned}
$$

Clearly $T_{n} \leqq n$ and $T_{n} \leqq T_{n+1}$. If $S=\lim _{n} T_{n}$ then $S$ is $F_{T_{n}}$-measurable for all $n$. The event $(S=\infty)$ is contained in $\bigcup_{n}\left(T_{n}>1\right)$ and so $(S=\infty)=\bigcup_{n}(S=\infty) \cap\left(T_{n}>1\right)$. Since $T_{n}$ increases the events $\left(T_{n}>1\right)$ are nondecreasing. It follows that $P(S=\infty)$ $=\lim P\left(S=\infty, T_{n}>1\right)$. Now $T_{n}>1$ implies $\left|Y_{T_{n}}\right| \geqq \varepsilon$. We have

$$
E\left(\left|Y_{T_{n}}\right|: S=\infty, T_{n}>1\right) \leqq E\left(\left|X_{T_{n}}\right|: S=\infty, T_{n}>1\right) \leqq E\left(\left|X_{T_{n}}\right|: S=\infty\right) .
$$

We deduce that

$$
\varepsilon P\left(S=\infty, T_{n}>1\right) \leqq E\left(\left|X_{T_{n}}\right|: S=\infty\right) .
$$

Let $n \rightarrow \infty$ to get $P(S=\infty)=0$. Finally note that $(S=\infty)=\left(\lim \sup \left|Y_{n}\right| \geqq \varepsilon\right)$.

REMARKS. 1. Martingale convergence theorem implies

$$
E\left(X_{\infty} \mid F_{n}\right) \rightarrow E\left(X_{\infty} \mid F_{\infty}\right) \text { almost surely. }
$$

Thus Lemma 3 shows that $E\left(X_{n} \mid F_{n}\right) \rightarrow E\left(X_{\infty} \mid F_{\infty}\right)$ almost surely. This will be used below.

2. The absence of an upcrossing inequality makes the following theorem interesting.

We have the following modification theorem.

THEOREM 4. Let $X_{t}$ be right continuous and uniformly bounded. Suppose $F_{t}$ is an increasing and right continuous family of $\sigma$-fields. Then there exists a right continuous modification of the stochastic process $E\left(X_{t} \mid F_{t}\right)$.

Proof. Assume $X_{t}$ is right continuous. Let $T$ be any stopping rule relative to $F_{t}$. Put $Y_{t}=E\left(X_{t} \mid F_{t}\right)$. We shall show below that the limit as $r \downarrow T, r$ running over the diadic rationals of $Y_{r}$ exists and equals $E\left(X_{T} \mid F_{T}\right)$. For this purpose we may assume that $E\left(X_{T} \mid F_{T}\right)=0$. Let $\varepsilon>0$ be given. Define a decreasing sequence $T_{n}$ of stopping rules by

$$
\begin{aligned}
T_{n} & =\inf \left(i / 2^{n}: i \geqq\left[2^{n} T\right]+1 \text { and } Y_{i / 2^{n}} \geqq \varepsilon\right), \\
& =\infty \quad \text { if there is no such } i,
\end{aligned}
$$

where [x] denotes the integral part of $x$. If $T_{n}<\infty$ then $Y_{T_{n}} \geqq \varepsilon$. Let $S=\lim T_{n}$. The event $(S=T)$ is identical with the event $\left(\lim \sup _{r>T ; r \rightarrow T} Y_{r} \geqq \varepsilon\right)$.

Since $\bigcap_{n} F_{T_{n}}=F_{S}\left[1, \mathrm{~T} 42\right.$, p. 67], Lemma 3 implies that $\lim Y_{T_{n}}$ exists and equals $E\left(X_{S} \mid F_{S}\right)$, almost surely. Thus

$$
\varepsilon P(S=T) \leqq E\left(\lim Y_{T_{n}}:(T=S)\right)=E\left(E\left(X_{S} \mid F_{S}\right):(T=S)\right) .
$$


The event $(S=T) \in F_{T} \subset F_{S}$. Hence

$$
\begin{aligned}
E\left(E\left(X_{S} \mid F_{S}\right): S=T\right) & =E\left(X_{S}: S=T\right) \\
& =E\left(X_{T}: S=T\right)=E\left(E\left(X_{T} \mid F_{T}\right): S=T\right) \\
& =0 \text { because } E\left(X_{T} \mid F_{T}\right)=0 \text { by assumption. }
\end{aligned}
$$

Thus $P\left(\lim \sup _{r>T ; r \downarrow T} Y_{r} \geqq \varepsilon\right)=0$ for all $\varepsilon>0$, i.e. $\lim \sup _{r>T ; r \rightarrow T} Y_{r}=0$. A similar reasoning shows that $\lim \inf _{r>T ; r \rightarrow T} Y_{r}=0$ almost surely. Thus with probability one $\lim _{r \downarrow T} Y_{r}$ exists and equals $E\left(X_{T} \mid F_{T}\right)$. Define the processes $U_{t}$ and $V_{t}$ by

$$
\begin{aligned}
U_{t} & =\limsup _{r \downarrow t} Y_{r}, \\
V_{t} & =\liminf _{r \downarrow t} Y_{r}, \quad r \text { diadic rational. }
\end{aligned}
$$

$U_{t}$ and $V_{t}$ are progressively measurable [1, D48, p. 70]. We also have

$$
U_{t} \geqq \lim _{s \downarrow t} \sup _{s}, \quad V_{t} \leqq \liminf _{s \downarrow t} V_{s} .
$$

Fix $\varepsilon>0$ and let the stopping rule $T$ be defined by

$$
\begin{aligned}
T & =\inf \left(t: U_{t}-V_{t} \geqq \varepsilon\right), \\
& =\infty \quad \text { if there is no such } t .
\end{aligned}
$$

If $T<\infty$ from what we said above $U_{T}-V_{T} \geqq \varepsilon$, i.e. that for the stopping rule $T$, $\lim \sup _{r \downarrow T} Y_{r} \geqq \varepsilon+\lim _{\inf _{r \downarrow T}} Y_{r}$. We have shown this cannot occur with positive probability. Thus except for a null set $\lim _{r \downarrow t} Y_{r}$ exists for all $t$. This limit then is the right continuous modification of $Y_{t}$ we were looking for. Q.E.D.

Now a natural question is whether we can require $Y_{t}$ to have left limits provided $X_{t}$ has left limits. The answer is yes. We need

Lemma 5. Let $X_{t}$ and $F_{t}$ be as in Theorem 4, and let $Y_{t}$ be a right continuous modification of $E\left(X_{t} \mid F_{t}\right)$. Then for any stopping rule $T$ we have $Y_{T}=E\left(X_{T} \mid F_{T}\right)$.

Proof. The proof is simple. If the stop rules $T_{n}$ are defined by $T_{n}=\left(\left[2^{n} T\right]+1\right) / 2^{n}$ where $[x]$ denotes the integral part of $x$, then $T_{n} \downarrow T$. By right continuity $Y_{T_{n}} \rightarrow Y_{T}$. Lemma 1 implies that $Y_{T_{n}}=E\left(X_{T_{n}} \mid F_{T_{n}}\right)$. Since $X_{T_{n}} \rightarrow X_{T}$ we may use Lemma 3 to complete proof.

Now we have

THEOREM 6. Let $X_{t}$ be right continuous, have left limits and be uniformly bounded. If $F_{t}$ is an increasing right continuous family of $\sigma$-fields then we can choose a right continuous with left limits modification of $E\left(X_{t} \mid F_{t}\right)$.

Proof. Let $Y_{t}$ be a right continuous modification of $E\left(X_{t} \mid F_{t}\right)$; this is guaranteed by Theorem 4 . We will show that $Y_{t}$ has left limits almost surely. Let $\varepsilon>0$ and define stopping rules $T_{n}$ as follows:

$$
\begin{aligned}
T_{1} & =\inf \left(t:\left|Y_{t}-Y_{0}\right| \geqq \varepsilon\right), \\
& =\infty \quad \text { if there is no such } t,
\end{aligned}
$$


and, in general,

$$
\begin{aligned}
T_{n+1} & =\inf \left(t: t \geqq T_{n},\left|Y_{t}-Y_{T_{n}}\right| \geqq \varepsilon\right), \\
& =\infty \quad \text { if there is no such } t .
\end{aligned}
$$

If $T_{n+1}<\infty$, by right continuity of $Y_{t}$ we have $\left|Y_{T_{n+1}}-Y_{T_{n}}\right| \geqq \varepsilon$. By Lemma 5 we have $Y_{T_{n}}=E\left(X_{T_{n}} \mid F_{T_{n}}\right)$. Since the sequence $T_{n}$ increases and $X_{t}$ has left limits $X_{T_{n}}$ converges on the set $\left(\lim T_{n}<\infty\right)$. We have

$$
\begin{aligned}
\varepsilon P\left(\lim T_{n}<\infty\right) & \leqq \varepsilon P\left(T_{n+1}<\infty\right) \\
& \leqq E\left(\left|Y_{T_{n+1}}-Y_{T_{n}}\right|: T_{n+1}<\infty\right) \leqq E\left(\left|X_{T_{n+1}}-X_{T_{n}}\right|: T_{n+1}<\infty\right) .
\end{aligned}
$$

Letting $n \rightarrow \infty$ we conclude that

$$
P\left(\lim T_{n}<\infty\right)=0 .
$$

Thus almost surely for each $\varepsilon>0$, there exists a sequence $T_{n}$ of stopping rules with $T_{n}<T_{n+1}, T_{n} \uparrow \infty$ such that the oscillation in the intervals $\left[T_{n}, T_{n+1}\right)$ is less than $2 \varepsilon$. This means that $Y_{t}$ has left limits almost surely. That finishes the proof.

2. In this section we develop some results that contain the modification theorem on super martingales. We assume that all processes considered are progressively measurable relative to a fixed increasing right continuous family $F_{t}$ of $\sigma$-fields. For simplicity we further assume that all processes are uniformly bounded. This restriction can easily be removed. If for the process $X(t), X(\infty)$ is not defined, simply define it to be zero.

THEOREM 7. Let $X(t)$ be a stochastic process with the following property:

If $T_{n}$ is a decreasing sequence of discrete valued stopping rules then $\lim X\left(T_{n}\right)$ exists almost surely.

Then except for a null set the limit $\lim _{r \downarrow t} X_{r}, r$ running over diadic rationals, exists for all $t$.

Proof. We will only indicate the proof since it is similar to the proof of Theorem 4. Let $T$ be any stopping rule. We will show that $\lim _{r \downarrow T} X(r), r$ running over diadic rationals, exists almost surely. If this were not the case there would exist numbers $b>a$ with

$$
\limsup _{r \downarrow T} X(r)>b>a>\liminf _{r \downarrow T} X(r)
$$

Define the stopping rules $T_{n}$ and $S_{n}$ as follows:

$$
\begin{aligned}
T_{n} & =\inf \left(i / 2^{n}: i \geqq\left[2^{n} T\right]+1 \text { and } X\left(i / 2^{n}\right)>b\right), \\
& =\infty \text { if there is no such } i ; \\
S_{n} & =\inf \left(j / 2^{n}: j \geqq\left[2^{n} T\right]+1 \text { and } X\left(j / 2^{n}\right)<a\right), \\
& =\infty \quad \text { if there is no such } j .
\end{aligned}
$$

Clearly $T_{n}$ and $S_{n}$ decrease. The set where lim $\sup _{r \downarrow t} X(r)>b>a>\lim \inf _{r \downarrow T} X(r)$ is identical with $\lim T_{n}=\lim S_{n}=T$. Call this set $A$. Choose small positive $\varepsilon_{n}$ with 
$\sum_{n} \varepsilon_{n}<P(A)$. Define a decreasing sequence of discrete valued stopping rules $U_{n}$ as follows: $U_{1}=T_{1}$. There exists an integer $n_{1}$ with

$$
P\left(A, T_{1} \geqq S_{n_{1}}\right)<\varepsilon_{1}, \quad \text { since } S_{n} \downarrow T \text { on } A .
$$

Put $U_{2}=U_{1} \wedge S_{n_{1}}$. There exists an $n_{2}$ with $P\left(A, U_{2} \geqq T_{n_{2}}\right)<\varepsilon_{2}$. Put $U_{3}=U_{2} \wedge T_{n_{2}}$. And so on. We thus get a decreasing sequence $U_{n}$ of stopping rules such that at least on a subset of $A$ of positive measure, $X\left(U_{2 n+1}\right)>b$ and $X\left(U_{2 n}\right)<a$, contradicting the assumption that $X\left(U_{n}\right)$ converges almost surely. Now define, as in Theorem 4,

$$
Y_{1}(t)=\limsup _{r \downarrow t} X(r), \quad Y_{2}(t)=\liminf _{r \downarrow t} X(r) .
$$

If $P\left(Y_{1}(t) \neq Y_{2}(t)\right.$ for some $\left.t\right)>0$, then there exist a stopping rule $T$ and $\varepsilon>0$ with $Y_{1}(T)>Y_{2}(T)+\varepsilon$ and this contradicts what we have already proved. This completes the proof.

REMARK. In Theorem 7 if $X(t)$ is a super martingale and we put $Y(t)=\lim _{r \downarrow t} X(r)$ then $Y(t)$ is right continuous. $Y(t)$ is $F_{t}$-measurable since $F_{t}$ is right continuous. This then implies the existence of right continuous modifications of measurable super martingales. The existence of left limits is no problem.

LEMMA 8. If $X(t)$ is a right continuous stochastic process such that for all increasing sequences $T_{n}$ of stopping times $\lim X\left(T_{n}\right)$ exists then $X(t)$ has left limits almost surely.

Proof. This is implicit in the proof of Theorem 6. Indeed if $\varepsilon>0$ and the stopping rules $T_{n}$ are defined by

$$
\begin{aligned}
T_{1} & =\inf \left(t:\left|X_{t}-X_{0}\right| \geqq \varepsilon\right), \\
& =\infty \quad \text { if there is no such } t ; \\
T_{n+1} & =\inf \left(t: t \geqq T_{n} \text { and }\left|X_{t}-X_{T_{n}}\right| \geqq \varepsilon\right), \\
& =\infty \quad \text { if there is no such } t,
\end{aligned}
$$

the assumption that $X_{T_{n}}$ converges implies that $T_{n} \uparrow \infty$ almost surely. It follows that $X(t)$ has left limits almost surely. Q.E.D.

Now suppose that $X(t)$ is a super martingale. Consider the processes

$$
X_{n}(t)=X\left((i+1) / 2^{n}\right) \text { if } i / 2^{n} \leqq t<(i+1) / 2^{n} .
$$

$X_{n}(t)$ are right continuous. Then there exists a right continuous modification $Y_{n}(t)$ of the process $E\left(X_{n}(t) \mid F_{t}\right)$. Since $X(t)$ is a super martingale $Y_{n}(t)$ is nondecreasing, i.e. $Y_{n}(t) \leqq Y_{n+1}(t)$. Put $Y(t)=\lim _{n \rightarrow \infty} Y_{n}(t)$. $Y(t)$ is clearly a measurable modification of $X(t)$. (Of course we assume that $E(X(t))$ is a right continuous function of $t$.) Theorem 7 and Lemma 8 say that $Y(t)$ has a right continuous with left limits modification. Since, for each $n, Y_{n}(t)$ is a super martingale, a result of Meyer $[1, \mathrm{~T}, \mathrm{p} .99]$ implies that $Y(t)$ is right continuous and has left limits. The following is a slight generalisation of the above mentioned result of Meyer. 
Lemma 9. Let $X(t)$ be a super martingale with the following properties:

1. $X(t)$ is progressively measurable and is lower semicontinuous from the right, i.e. $X(t) \leqq \lim \inf _{s>t ; s \downarrow t} X(s)$.

2. For stopping times $T$ and $S$ with $T \leqq S$ we have $E(X(T)) \geqq E(X(S))$.

Then $X(t)$ is right continuous and has left limits almost surely.

Proof. If $T_{n}$ are decreasing, discrete valued stopping rules, $X\left(T_{n}\right)$ converges by the super martingale convergence theorem. By Theorem 7, except for a null set, $Y_{t}=\lim _{r \downarrow t} X(r)$ exists for all $t$. Lower semicontinuity on the right implies that $Y(t) \geqq X(t)$ for all $t$.

For each stopping time $T$ we get

$$
Y(T)=\lim _{r>T: r \downarrow T} X(r)=\lim X\left(\frac{\left[2^{n} T\right]+1}{2^{n}}\right)
$$

so that

$$
E(Y(T))=\lim E\left(X\left(\frac{\left[2^{n} T\right]+1}{2^{n}}\right)\right) \leqq E(X(T))
$$

because $\left(\left[2^{n} T\right]+1\right) / 2^{n}$ being a stopping rule $>T$ we have

$$
E\left(X\left(\frac{\left[2^{n} T\right]+1}{2^{n}}\right)\right) \leqq E(X(T)) .
$$

Thus for every stopping rule $T, X(T)=Y(T)$ almost surely. If $\varepsilon>0$ and

$$
\begin{aligned}
T & =\inf \left(t: Y_{t}-X_{t} \geqq \varepsilon\right), \\
& =\infty \quad \text { if there is no such } t,
\end{aligned}
$$

then by right continuity of $Y_{t}$ and lower semicontinuity of $X_{t}$ from the right we must have $Y_{T}-X_{T} \geqq \varepsilon$ on the set $(T<\infty)$. But this contradicts $Y_{T}=X_{T}$ for all stopping rules. Thus

$$
P\left(Y_{t}=X_{t} \text { for all } t\right)=1,
$$

i.e. $X_{t}$ is almost surely right continuous and by Lemma 8 has left limits. That completes the proof.

REMARK. Let us indicate to what extent the results of $\S \S 1$ and 2 generalise to Banach space valued variables. The conditional expectation of a variable $X$ relative to a sub- $\sigma$-field $G$ will be defined as in [2, p. 22]. The proofs of Lemmas 1, 2 and 3 are generalised verbatim. Theorem 4 needs slight changes. Assume as in Theorem 4 that $E\left(X_{T} \mid F_{T}\right)=0$. Define

$$
\begin{aligned}
T_{n} & =\inf \left(i / 2^{n}: i \geqq\left[2^{n} T\right]+1 \text { and }\left|Y_{i / 2^{n}}\right| \geqq \varepsilon\right) \\
& =\infty \text { if there is no such } i .
\end{aligned}
$$

Put $S_{n}=\left(\left[2^{n} T\right]+1\right) / 2^{n}$. If

$$
P\left(\limsup _{r>T ; r \downarrow T}\left|Y_{r}\right| \geqq \varepsilon\right)>0
$$


we can always choose indices $k_{n}$ such that $P\left(T_{n}<S_{k_{n}}\right.$ for all $\left.n\right)>0$. Define $U_{n}=S_{k_{n}}$ $\wedge T_{n} . U_{n} \downarrow T$ so that $Y_{U_{n}}$ tends to $E\left(X_{T} / F_{T}\right)=0$. This is not possible on the set $\left(U_{n}<S_{k_{n}}\right.$ for all $\left.n\right)$. Now let

$$
Z_{t}=\lim _{r, s>t ; r, s \downarrow t} \sup _{t}\left|Y_{r}-Y_{s}\right| .
$$

$Z_{t}$ is progressively measurable and $Z_{T}=0$ for all stopping rules $T$. It follows that $P\left(Z_{t}>0\right.$ for some $\left.t\right)=0$.

Lemma 5 and Theorem 6 do not need any changes. The changes needed in the proof of Theorem 7 parallel those that we have already indicated in the case of Theorem 4.

AdDED IN PROOF. ACKNOWLEDGEMENT OF PRIORITY. P. A. Meyer and F. Mertens have obtained this result earlier than the author.

\section{REFERENCES}

1. P. A. Meyer, Probability and potentials, Blaisdell, Waltham, Mass., 1966. MR 34 \#5119.

2. S. D. Chatterji, Martingale convergence and the Radon-Nikodym theorem in Banach spaces, Math. Scand. 22 (1968), 21-41. MR 39 \#7645.

Matematisk Institut, Aarhus Universitets, Universitetsparken, 8000 Aarhus C., DANMARK 